\title{
Expression of mTOR/70S6K signaling pathway in pathological scar fibroblasts and the effects of resveratrol intervention
}

\author{
ZHI-MING TANG ${ }^{1}$, XIAO-XIANG ZHAI ${ }^{1}$ and JI-CUN DING $^{2}$ \\ ${ }^{1}$ Department of Dermatology, Xuzhou Hospital of Traditional Chinese Medicine, Xuzhou, Jiangsu 221003; \\ ${ }^{2}$ Department of Burns and Plastic Surgery, The Affiliated Xuzhou Center Hospital of Nanjing University \\ of Chinese Medicine, Xuzhou, Jiangsu 221009, P.R. China
}

Received August 22, 2016; Accepted November 29, 2016

DOI: $10.3892 / \mathrm{mmr} .2017 .6339$

\begin{abstract}
The aim of the study was to examine the expression of mammalian target of rapamycin (mTOR)/70S6K signaling pathway in pathological scar fibroblasts and the effects of resveratrol (Res) intervention. The mTOR and 70S6K in pathological scar and normal skin fibroblasts were detected by immunofluorescence following treatment with different concentrations of Res. RT-PCR and western blot analysis were used to detect the expression of mTOR and 70S6K mRNA and protein, respectively. Immunofluorescence showed that the expression of 70S6K and mTOR was significantly enhanced in pathological scar fibroblasts, and mainly expressed in the nucleus, but not in normal skin fibroblasts. RT-PCR and western blot analysis showed that after different concentrations of Res treatments, the mTOR and 70S6K mRNA and protein expression significantly $(\mathrm{P}<0.05)$ decreased in a dose-dependent manner. In conclusion, the expression of mTOR/70S6K signaling pathway in pathological scar fibroblasts was significantly enhanced. Res can downregulate the expression of mTOR and 70S6K to achieve the inhibition of pathological scar fibroblast proliferation.
\end{abstract}

\section{Introduction}

Pathological scar is a hyperplastic disease of connective tissue in the skin. At present, many studies in China and other countries have focused on its pathogenesis, which has yet to be completely elucidated, and no breakthrough has been obtained for the prevention and treatment of pathological scar. The excessive proliferation of fibroblasts plays a critical role in the formation process of pathological scar. As a polyphenol compound, resveratrol (Res) has an inhibitory effect on the

Correspondence to: Dr Ji-Cun Ding, Department of Burns and Plastic Surgery, The Affiliated Xuzhou Center Hospital of Nanjing University of Chinese Medicine, 199 South Jiefang Road, Xuzhou, Jiangsu 221009, P.R. China

E-mail: ding_jicun1@163.com

Key words: resveratrol, pathological scar, fibroblasts, mammalian target of rapamycin/70S6K signaling pathway proliferation of various tumor cells (1). In a previous study, we showed that Res inhibited the proliferation of pathological scar fibroblasts (2), although the specific mechanism has not been completely clarified. However, pathological scar had tumor-like characteristics. Various studies have shown that many tumor-related genes are involved in the formation of pathological scar $(3,4)$, indicating the great significance of studying pathological scar. Mammalian target of rapamycin (mTOR) gene was closely related to the abnormal proliferation of cells and the occurrence of tumor and markedly enhanced its expression in various tumors, including glioma, esophageal and colorectal cancer (5). Therefore, the mTOR signaling pathway may be important in the process of the proliferation of pathological scar fibroblasts.

Therefore, the present study was undertaken to clarify the important role that mTOR plays in the signaling pathway of pathological scar fibroblasts and to determine the effects of Res intervention. Immunofluorescence, RT-PCR, western blot analysis and other technologies were respectively used to detect the expression of mTOR and its downstream molecule $70 \mathrm{~S} 6 \mathrm{~K}$ in pathological scar fibroblasts and the effects of Res in order to clarify and provide a theoretical basis for the application of Res in the clinical treatment of pathological scar.

\section{Materials and methods}

\section{Materials}

Main reagents. The main reagents used in the study were: Fetal bovine serum (FBS; Hangzhou Sijiqing Biological Engineering Material Co., Ltd., Hangzhou, China), Dulbecco's modified Eagle's medium (DMEM) and phosphate-buffered saline (PBS) solution (Tianjin Hao Yang Biological Technology Co., Ltd., Tianjin, China), D-Hank's liquid (Beijing Hua Maike Biotechnology Co., Ltd., Beijing, China), $0.25 \%$ trypsin-EDTA solution (Beijing Solarbio Technology Co., Ltd., Beijing, China), TRIzol reagent and reverse transcription kit superscript III (both from Invitrogen Life Technologies, Carlsbad, CA, USA), rabbit anti-human mTOR, 70S6K monoclonal antibody (Santa Cruz Biotechnology, Inc., Santa Cruz, CA, USA), goat anti-rabbit IgG second antibody (BD Pharmingen, San Diego, CA, USA) labeled by fluorescein isothiocyanate (FITC), and Res (Xi'an Rui Ying Biological Technology Co., Ltd., Xi'an, China). 
Main instruments. High-speed centrifuge (Changsha Pingfan Instrument Co., Ltd., Changsha, China), $\mathrm{CO}_{2}$ incubator (Sanyo, Tokyo, Japan), electrophoresis apparatus (Beijing Liuyi Instrument Factory, Beijing, China), PCR instrument (Shanghai Shan Fu Bio-Tech Co., Ltd., Shanghai, China), Quantity One gel image-forming analysis system (Bio-Rad, Berkeley, CA, USA), fluorescence microscope and digital CCD imaging device (Olympus, Tokyo, Japan), Image-Pro Plus imaging analysis system (Media Cybernetics, Inc., Rockville, MD, USA), and Odyssey infrared fluorescence imaging instrument (LI-COR Biosciences, Lincoln, NE, USA) were used in the present study.

Specimen source. Experimental specimens were derived from patients with pathological scar surgery in the Departments of Burns and Plastic Surgery, and Dermatology (Jiangsu, China). The specimens were obtained from the patients with signed informed consent.

\section{Methods}

Separation and culture of fibroblast cells. Tissue specimens of pathological scar and normal skin near the scar were cut respectively, under aseptic conditions, and epithelial and subcutaneous tissues were removed carefully with ophthalmic scissors. The specimens were washed three times with D-Hank's liquid and sheared into tissue masses of $1 \mathrm{~mm}^{3}$. The specimens were placed in a sterile bottle, filtered after being digested into cotton-shape with $20 \%$ trypsin-EDTA solution and centrifuged at $650 \mathrm{x}$ g for $5 \mathrm{~min}$, and $20 \%$ DMEM liquid of $5 \mathrm{ml}$ containing FBS mixture was added to deposit cells. The specimens were placed in a culture bottle of $25 \mathrm{ml}$ to cultivate and were cultivated in a $5 \% \mathrm{CO}_{2}$ incubator at $37^{\circ} \mathrm{C}$. The liquid was replaced by $20 \%$ DMEM liquid containing FBS, and growth was observed under microscope (Olympus Corp.). When the cell growth was close to completion, the passage was conducted with a proportion of $1: 3$.

Expression of mTOR and 70S6K in fibroblast cells detected by immunofluorescence. Fifth generation-cultured fibroblast cells were taken, $4 \%$ paraformaldehyde was added, and fibroblast cells were fixed for $30 \mathrm{~min}$ at $25^{\circ} \mathrm{C}$, and washed three times with PBS buffer liquid. The cells were then incubated for 20 min with $0.1 \%$ Triton $\mathrm{X}-100$ at $37^{\circ} \mathrm{C}$, washed with PBS liquid, and sealed for $1 \mathrm{~h}$ with non-immune goat serum. When the serum was absorbed completely, primary antibody was added. Rabbit anti-human mTOR monoclonal antibody (dilution: 1:500; catalog no. 8665T) and rabbit anti-human 70S6K monoclonal antibody (dilution: 1:500; catalog no. 2903T) were obtained from Cell Signaling Technology (Danvers, MA, USA). The cells were incubated overnight at $4^{\circ} \mathrm{C}$, washed with PBS liquid the following day, and FITC-labeled goat anti-rabbit secondary antibody (dilution: 1:200; catalog no. 4412S; Cell Signaling Technology) was added. Then they were incubated for $60 \mathrm{~min}$ at $37^{\circ} \mathrm{C}$, washed with PBS liquid, redyed with DAPI in the dark, and observed under a fluorescence microscope (Olympus Corp.) after $10 \mathrm{~min}$.

Expression of mTOR, 70S6K in pathological scar fibroblast cells detected by RT-PCR. Vaccination cells in 96-well culture plate were divided into the control group (group A) and
Table I. mTOR and 70S6K gene primer sequences and amplification fragment length of the product.

\begin{tabular}{llc}
\hline Gene & \multicolumn{1}{c}{ Primer sequences (5'-3') } & $\begin{array}{c}\text { Product } \\
\text { size (bp) }\end{array}$ \\
\hline mTOR & $\begin{array}{l}\text { U: ACTCGCTTCTATGACCAACTGA } \\
\text { D: TTTCCATGACAACTGGGTCATTG }\end{array}$ & 494 \\
70S6K & U: TACTTCGGGTACTTGGTAA & 604 \\
& D: GATGAAGGGATGCTTTACT & \\
\hline
\end{tabular}

mTOR, mammalian target of rapamycin. U, upstream sequence; $\mathrm{D}$, downstream sequence.

3 drug concentration groups: group B (at a concentration of $10 \mu \mathrm{mol} / \mathrm{l}$ ), group C (of $50 \mu \mathrm{mol} / 1$ ) and group D (of $100 \mu \mathrm{mol} / \mathrm{l}$ ), (concentration screening was obtained in accordance with Res concentration when the $\mathrm{IC}_{50}$ of cells in the experiment ranging from $1 / 3$ to $2 / 3$ ) with 24 culture well in each group. The 5 th generation of fibroblast cells was transformed into single cell suspension with DMEM solution containing 10\% FBS, vaccinated in 96-well culture plate with a density of $1.0 \times 10^{4} / \mathrm{ml}$ with $200 \mu \mathrm{l}$ per well, placed into $\mathrm{CO}_{2}$ incubator for routine culture. When cells adhered, the old culture solution in the well of the culture plate was absorbed, and DMEM solution of $180 \mu \mathrm{l}$ containing no FBS was added to each well, followed by adding corresponding concentration of Res $20 \mu \mathrm{l}$ into the 3 groups, and DMEM solution $(20 \mu \mathrm{l})$ containing $10 \%$ FBS was added into the control group.

After incubating for $48 \mathrm{~h}$ under the standard environment, a TRIzol reagent total RNA extraction kit was used to extract RNA, and the procedures were in strict accordance with the instructions. Electrophoresis was used to determine the integrity of RNA extraction. Through measurement, $\mathrm{A}_{260} / \mathrm{A}_{280}$ (the proportion of RNA light absorption value at 260 and $280 \mathrm{~nm}$ ) values of all the samples were $>1.8$, which indicated that sample purification was high without obvious protein contamination. Total RNA purity was regulated uniformly into $0.5 \mathrm{~g} / 1$ and preserved at $-70^{\circ} \mathrm{C}$.

RNA $(6 \mu \mathrm{l})$ was taken as reverse transcription template whose reaction system was $20 \mu 1$, and the reaction condition was $37^{\circ} \mathrm{C} 1 \mathrm{~h}, 95^{\circ} \mathrm{C} 3 \mathrm{~min}$. Standard product of cDNA template was prepared by standard curve quantitative method and diluted with 10-fold gradient in turn to prepare 5-fold gradients cDNA template quantitative standard product. mRNA list of gene was obtained from GenBank database, and Primer Express software (Applied Biosystems, Foster City, CA, USA) was used, with specific primers being designed in CDS area. Table I shows the primer sequences.

The reaction system of PCA was $50 \mu 1$, and reaction conditions were: $3 \mathrm{~min}$ initial denaturation at $93^{\circ} \mathrm{C}, 30 \mathrm{sec}$ denaturation at $93^{\circ} \mathrm{C}, 30 \mathrm{sec}$ annealing at $57^{\circ} \mathrm{C}$, repeated for 30 cycles, with a 5 min extension at $72^{\circ} \mathrm{C} ; 6 \mu$ l PCR product was taken for electrophoresis in $1.5 \%$ agarose gel. Quantity One gel imaging analysis system was used to analyze the grey value of each band, and the relative expression level of mRNA was expressed by the ratio grey value of target gene to that of reference gene $\beta$-actin grey. 

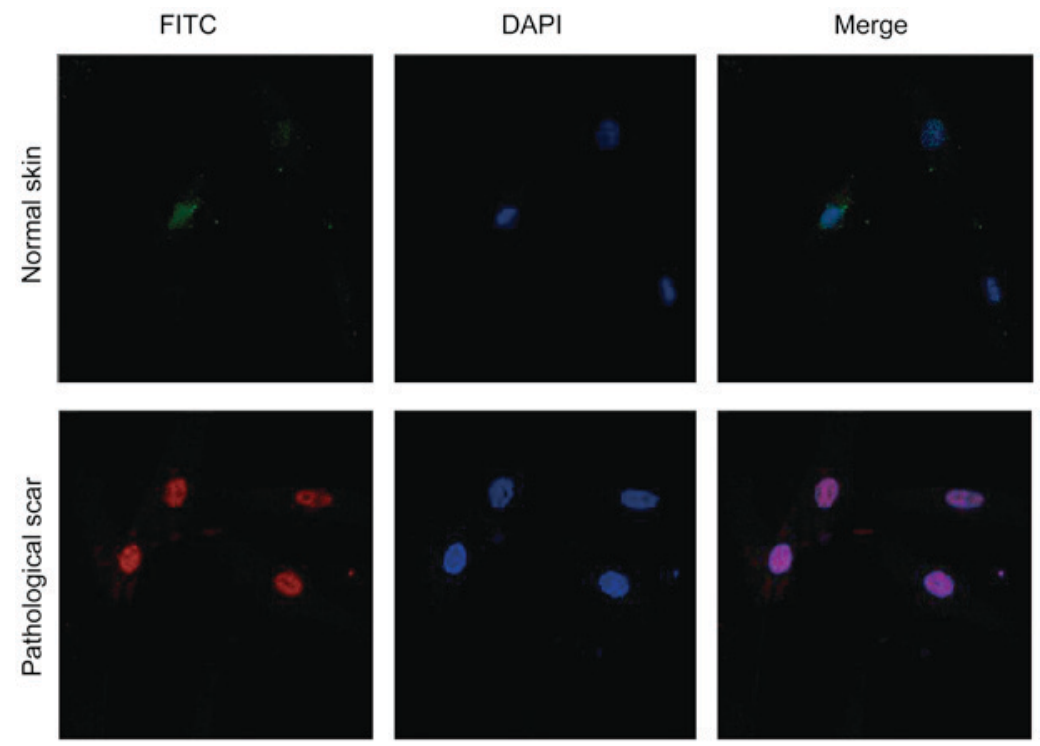

Figure 1. mTOR expression in fibroblast cells isolated from normal skin and pathological scar.
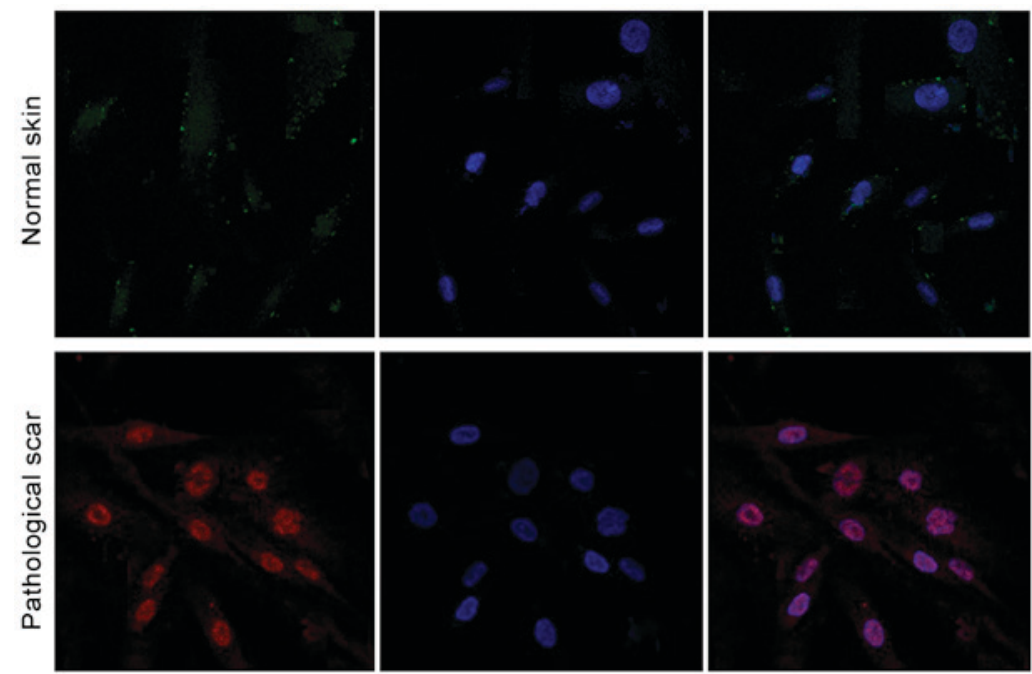

Figure 2. 70S6K expression in fibroblast cells isolated from normal skin and pathological scar.

Expression of mTOR and 70S6K protein in pathological scar fibroblast cells detected by western blot analysis. Cell culture and intervention methods were the same as the above. Cultured fibroblast cells were collected, washed twice with pre-cooled PBS, cell lysates were added, and the cells were placed on ice for 10-20 min, after being scraped with a cell homogenate. The cells were then centrifuged at at $8,500 \mathrm{x}$ g for $20 \mathrm{~min}$ under $4^{\circ} \mathrm{C}$ and the supernatant was removed. The Lowry method was used for supernatant protein quantification, $50 \mu \mathrm{g}$ protein was taken to $15 \%$ SDS-polyacrylamide gel electrophoresis. When bromophenol blue entered the bottom of the gel, protein was re-imprinted on the nitrocellulose filter, and the primary antibody was added for inclubation overnight at $4^{\circ} \mathrm{C}$. Corresponding secondary antibody labeled by horseradish peroxidase (HRP) was added, and the cells were incubated for $1 \mathrm{~h}$ at room temperature. After the membrane was washed, Odyssey infrared fluorescence imaging was used to scan them and form imaging, which was analyzed by Image-Pro Plus imaging analysis system.
Statistical analysis. SPSS 13.0 software (SPSS, Inc., Chicago, IL, USA) was used for data analysis. Measurement data were presented as the mean \pm standard deviation (mean \pm SD). PSS homogeneity of variance was tested by t-test. When variance was not homogeneous, the rank sum test was used. Pearson's method was used for correlation analysis. $\mathrm{P}<0.05$ was considered to indicate a statistically significant difference.

\section{Results}

Immunofluorescence results. The immunofluorescence detection result of cells showed that, mTOR was marked in red (Fig. 1). DAPI was marked in blue fluorescence in the nucleus (Fig. 1). The merged image (Fig. 1) indicated that mTOR expressed in the nucleus, while no obvious expression was found in fibroblast cells of the normal skin tissue (Fig. 1). Immunofluorescence detection results showed that, $70 \mathrm{~S} 6 \mathrm{~K}$ was expressed in the nucleus in pathological scar fibroblast cells (Fig. 2), while no obvious expression was found in normal skin tissue (Fig. 2). 
Table II. Relative expression of mTOR and 70S6K mRNA of various groups.

\begin{tabular}{lll}
\hline Groups & mTOR & $70 \mathrm{~S} 6 \mathrm{~K}$ \\
\hline A & $9.54 \pm 0.23$ & $8.71 \pm 0.21$ \\
B & $7.48 \pm 0.19^{\mathrm{a}}$ & $6.69 \pm 0.17^{\mathrm{a}}$ \\
C & $5.91 \pm 0.14^{\mathrm{b}}$ & $4.27 \pm 0.13^{\mathrm{b}}$ \\
$\mathrm{D}$ & $2.11 \pm 0.09^{\mathrm{c}}$ & $3.02 \pm 0.10^{\mathrm{c}}$ \\
\hline
\end{tabular}

${ }^{\mathrm{a}-\mathrm{c}} \mathrm{P}<0.05$ vs. group A using t-test $\left({ }^{\mathrm{a}} \mathrm{t}=2.14,2.18 ;{ }^{\mathrm{b}} \mathrm{t}=2.23,2.35 ;{ }^{\mathrm{c}} \mathrm{t}=2.43\right.$, 2.53). mTOR, mammalian target of rapamycin.

A Control $\frac{\text { Res }}{10 \mu \mathrm{mol} / / \mathrm{l} 50 \mu \mathrm{mol} / / 100 \mu \mathrm{mol} / \mathrm{I}}$

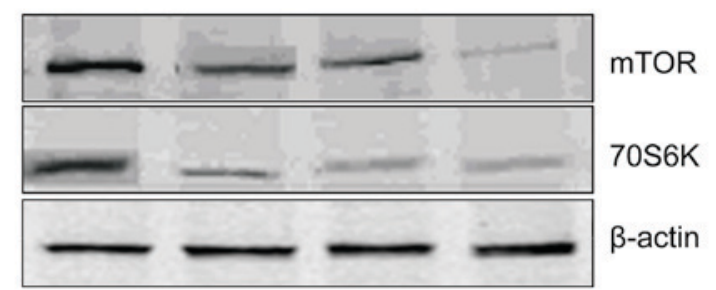

B

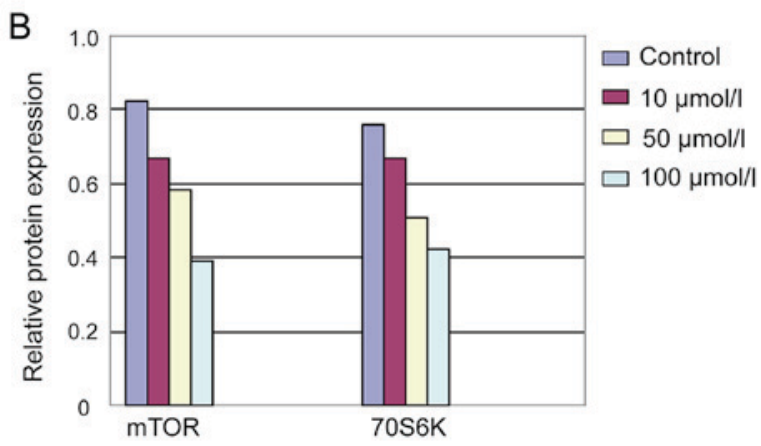

Figure 3. Expression of mTOR and 70S6K in pathological scar fibroblast cells after treatment with different concentrations of Res. (A) mTOR and 70S6K detected by western blot analysis. (B) Quantitative analysis of mTOR and 70S6K in different groups. mTOR, mammalian target of rapamycin.

RT-PCR results. After pathological scar fibroblast cells were intervened by Res concentrations of 10,50 and $100 \mu \mathrm{mol} / 1$, the mTOR and 70S6K mRNA relative expression levels decreased compared with those of the control group. The differences were statistically significant $(\mathrm{P}<0.05)$ (Table II). Correlation analysis using Pearson's method indicated that, relative expression of mTOR and 70S6K mRNA was negative with Res intervention concentration $(r=-0.872, \mathrm{P}=0.02 ; \mathrm{r}=-0.814$, $\mathrm{P}=0.03$ ).

Western blot analysis results. After pathological scar fibroblast cells were intervened by Res, the protein band ratio of mTOR and $70 \mathrm{~S} 6 \mathrm{~K}$ in the various concentration groups decreased significantly compared with that of the control group (Fig. 3). The analysis of Image-Pro Plus imaging analysis system showed that, relative expression of mTOR and 70S6K in the various concentration groups was significantly lower than those of the control group. The differences were statistically significant $(\mathrm{P}<0.05)$ (Fig. 3 and Table III).
Table III. Relative expression of mTOR, 70S6K protein of various groups.

\begin{tabular}{lcc}
\hline Groups & mTOR & \multicolumn{1}{c}{$70 \mathrm{~S} 6 \mathrm{~K}$} \\
\hline $\mathrm{A}$ & $0.825 \pm 0.073$ & $0.761 \pm 0.068$ \\
$\mathrm{~B}$ & $0.67 \pm 0.068^{\mathrm{a}}$ & $0.678 \pm 0.061^{\mathrm{a}}$ \\
$\mathrm{C}$ & $0.586 \pm 0.062^{\mathrm{b}}$ & $0.515 \pm 0.054^{\mathrm{b}}$ \\
$\mathrm{D}$ & $0.395 \pm 0.041^{\mathrm{c}}$ & $0.428 \pm 0.036^{\mathrm{c}}$ \\
\hline
\end{tabular}

${ }^{\mathrm{a}-\mathrm{c}} \mathrm{P}<0.05$ vs. group A using t-test $\left({ }^{\mathrm{a}} \mathrm{t}=1.93,2.04 ;{ }^{\mathrm{b}} \mathrm{t}=2.12,2.31 ;{ }^{\mathrm{c}} \mathrm{t}=2.46\right.$, 2.53). mTOR, mammalian target of rapamycin.

\section{Discussion}

Pathological scar includes hypertrophic scar and keloid. As typical fiber hyperplastic disease, pathological scar is the pathological product formed by the abnormal proliferation during the process of wound repair and healing and its pathological changes are mainly characterized by massive proliferation of fibroblasts and excessive deposition of extracellular matrix dominated by collagen (6). At present, there are many studies on pathological scar in China and other countries (7-9). However, its exact pathogenesis remains to be elucidated and no breakthrough has been obtained for its prevention and treatment. Despite the appearance of many therapeutic methods, the curative effects are unsatisfactory. Therefore, it is of vital practical significance to discuss the pathogenesis of pathological scar from a new perspective and seek safe and effective therapeutic methods and drugs

The pathogenesis of pathological scar remains complex. The excessive proliferation of fibroblasts and its increasing activity play a critical role in its formation. The excessive proliferation of pathological scar fibroblasts is the result of the integrated response of cells to the stimulation of various extracellular signals $(10,11)$. Since this integrated response is complete in cells, it inevitably involves the process of cell signal transduction. At present, more studies have focused on typical TGF- $\beta 1 /$ Smads signal pathway. Nevertheless, the specific mechanism of the proliferation of pathological scar fibroblasts was not revealed completely. Since the proliferation of pathological scar fibroblasts involved many multiple genes and multiple signal pathways, this process is extremely complex. In recent years, an increasing number of studies have shown that many types of independent Smad pathways, in addition to the typical Smad pathway, were also involved in the transduction process of TGF- $\beta 1$ signals. Some of them directly or indirectly were involved in tumor formation and were closely related to proliferation, invasion and transfer of tumor (12-15).

As the main signal pathway to regulate and control protein synthesis, mTOR signal pathway is involved in cell proliferation, differentiation and other regulations, making it a research hotspot, especially with regard to increasingly in-depth studies on tumor (16-18). Activated mTOR can act on downstream target protein, ribosomal protein S6 kinase $(70 \mathrm{~S} 6 \mathrm{~K})$, while $70 \mathrm{~S} 6 \mathrm{~K}$ is the key regulating factor of protein translation (19-21). 
Studies in recent years have demonstrated that mTOR gene was closely related to abnormal proliferation of cells and the occurrence of tumor and markedly enhanced its expression in various tumors, including glioma, esophageal cancer and colorectal cancer $(16,18)$. Pathologically, pathological scar belongs to benign solid tumor and can grow to surrounding normal tissues invasively and its pathological characteristics are very similar to those of tumor, with tumor-like characteristics. This is an indication that mTOR signal pathway should also play an important role in the process of the proliferation of pathological scar fibroblasts.

Traditional Chinese medicine has a long history in the prevention and treatment of scars. The present research group has been engaged in the study of prevention and treatment of Chinese medicine on pathological scar and the undertaken subjects are all related to Res. As a non-flavonoid polyphenolic compound existing in Polygonum Cuspidatum, Veratrum nigrum, Cassia tora and other plants, Res belongs to a phytoalexin generated by plants in resistance to external stimulations, such as ultraviolet ray, fungus, virus infection and mechanical injury (22). It has multiple biological activities and pharmacological action, such as anti-inflammatory, anti-shock, antioxidation, inhibition of platelet aggregation, improving microcirculation, protection of mitochondrial functions and estrogenic effects (23-25). Our preliminary study also proved that Res inhibited the proliferation of pathological scar fibroblasts, but the specific mechanism of action has not been elucidated completely. It remains to be determined whether Res exerts an effect on the proliferation of pathological scar fibroblasts by regulating and controlling the mTOR/70S6K signal pathway. At present, there is no domestic and foreign literature to support this hypothesis. Therefore, our research group implemented some explorations on it. The present study applied cellular immunofluorescence to detect the expression of mTOR and 70S6K and found a clearly strengthened expression of mTOR and 70S6K in pathological scar fibroblasts, mainly in the cell nucleus, without obvious expression in fibroblasts of normal skin tissue. The results of RT-PCR and western blot analysis detection revealed that after pathological scar fibroblasts being intervened by Res with different concentrations, the expression of mTOR and 70S6K mRNA and protein decreased as compared with that of the control group, with statistically significant differences $(\mathrm{P}<0.05)$. Correlation analysis using the Pearson's method showed that the expression of mTOR and 70S6K mRNA showed a negative relationship with the intervention concentration of Res. This showed that the mTOR/70S6K pathway was involved in the formation of pathological scar, while Res inhibited the proliferation of pathological scar fibroblasts, which may be associated with reduction of the expression of mTOR and 70S6K.

\section{References}

1. Tinhofer I, Bernhard D, Senfter M, Anether G, Loeffler M, Kroemer G, Kofler R, Csordas A and Greil R: Resveratrol, a tumor-suppressive compound from grapes, induces apoptosis via a novel mitochondrial pathway controlled by Bcl-2. FASEB J 15 : $1613-1615,2001$.

2. Ding J, Zai X and Tang Z: Effects of resveratrol on fibroblasts of pathological scar and its Tgf- $\beta 1 /$ smads signaling pathway. J Hebei Med Univ 35: 37-41, 2014 (In Chinese).
3. Huang LP, Mao Z, Zhang L, Liu XX, Huang C and Jia ZS: Screening of differentially expressed genes in pathological scar tissues using expression microarray. Genet Mol Res 14: 10743-10751, 2015

4. Teofoli P, Barduagni S, Ribuffo M, Campanella A, De Pita' O and Puddu P: Expression of Bcl-2, p53, c-jun and c-fos protooncogenes in keloids and hypertrophic scars. J Dermatol Sci 22: 31-37, 1999.

5. Steelman LS, Chappell WH, Abrams SL, Kempf RC, Long J, Laidler P, Mijatovic S, Maksimovic-Ivanic D, Stivala F, Mazzarino MC, et al: Roles of the Raf/MEK/ERK and $\mathrm{PI} 3 \mathrm{~K} / \mathrm{PTEN} / \mathrm{Akt} / \mathrm{mTOR}$ pathways in controlling growth and sensitivity to therapy-implications for cancer and aging. Aging (Albany NY) 3: 192-222, 2011.

6. van der Veer WM, Bloemen MC, Ulrich MM, Molema G, van Zuijlen PP, Middelkoop E and Niessen FB: Potential cellular and molecular causes of hypertrophic scar formation. Burns 35: 15-29, 2009.

7. Bollero D, Malvasio V, Catalano F and Stella M: Negative pressure surgical management after pathological scar surgical excision: a first report. Int Wound J 12: 17-21, 2015.

8. Huang $\mathrm{C}$ and Ogawa R: The link between hypertension and pathological scarring: does hypertension cause or promote keloid and hypertrophic scar pathogenesis? Wound Repair Regen 22: 462-466, 2014

9. Zhai XX, Ding JC and Tang ZM: Resveratrol inhibits proliferation and induces apoptosis of pathological scar fibroblasts through the mechanism involving TGF- $\beta 1 /$ Smads signaling pathway. Cell Biochem Biophys 71: 1267-1272, 2015.

10. Susami T: Wound healing in the palatal mucosa and the mechanism of scar formation. Kokubyo Gakkai Zasshi 52: 596, 1985 (In Japanese).

11. Lu SL: Mechanism of scar formation and strategy of treatment. Zhonghua Shao Shang Za Zhi 29: 130-133, 2013 (In Chinese).

12. Sun Q, Guo S, Wang CC, Sun X, Wang D, Xu N, Jin SF and Li KZ: Cross-talk between TGF- $\beta /$ Smad pathway and Wnt/ $\beta$-catenin pathway in pathological scar formation. Int J Clin Exp Pathol 8: 7631-7639, 2015

13. Chen W, Fu X, Sun T, Sun X, Zhao Z and Sheng Z: Change of gene expression of transforming growth factor-beta1, Smad 2 and Smad 3 in hypertrophic scars skins. Zhonghua Wai Ke Za Zhi 40: 17-19, 2002 (In Chinese).

14. Brown KA, Pietenpol JA and Moses HL: A tale of two proteins: differential roles and regulation of Smad2 and Smad3 in TGF-beta signaling. J Cell Biochem 101: 9-33, 2007.

15. Yu R and Cen Y: Transforming growth factor beta1/Smad3 signal transduction pathway and post-traumatic scar formation. Zhongguo Xiu Fu Chong Jian Wai Ke Za Zhi 26: 330-335, 2012 (In Chinese).

16. Zhang YJ, Dai Q, Sun DF, Xiong H, Tian XQ, Gao FH, Xu MH, Chen GQ, Han ZG and Fang JY: mTOR signaling pathway is a target for the treatment of colorectal cancer. Ann Surg Oncol 16: 2617-2628, 2009.

17. Laplante M and Sabatini DM: mTOR signaling in growth control and disease. Cell 149: 274-293, 2012.

18. Petroulakis E, Mamane Y, Le Bacquer O, Shahbazian D and Sonenberg N: mTOR signaling: implications for cancer and anticancer therapy. Br J Cancer 96 (Suppl): R11-R15, 2007.

19. Shinojima N, Yokoyama T, Kondo Y and Kondo S: Roles of the Akt/mTOR/p70S6K and ERK1/2 signaling pathways in curcumin-induced autophagy. Autophagy 3: 635-637, 2007.

20. Heinonen H, Nieminen A, Saarela M, Kallioniemi A, Klefström J, Hautaniemi S and Monni O: Deciphering downstream gene targets of $\mathrm{PI} 3 \mathrm{~K} / \mathrm{mTOR} / \mathrm{p} 70 \mathrm{~S} 6 \mathrm{~K}$ pathway in breast cancer. BMC Genomics 9: 348, 2008 .

21. Hou G, Xue L, Lu Z, Fan T, Tian F and Xue Y: An activated mTOR/p70S6K signaling pathway in esophageal squamous cell carcinoma cell lines and inhibition of the pathway by rapamycin and siRNA against mTOR. Cancer Lett 253: 236-248, 2007.

22. Zhao KS: Biological characteristics and effects of resveratrol. Chinese Journal of Pathophysiology 28: 1709-1711 2012 (In Chinese).

23. Pangeni R, Sahni JK, Ali J, Sharma S and Baboota S: Resveratrol: review on therapeutic potential and recent advances in drug delivery. Expert Opin Drug Deliv 11: 1285-1298, 2014.

24. Wu JM, Hsieh TC and Wang Z: Cardioprotection by resveratrol: a review of effects/targets in cultured cells and animal tissues. Am J Cardiovasc Dis 1: 38-47, 2011.

25. Athar M, Back JH, Tang X, Kim KH, Kopelovich L, Bickers DR and Kim AL: Resveratrol: a review of preclinical studies for human cancer prevention. Toxicol Appl Pharmacol 224: 274-283, 2007. 